\title{
SOME REMARKS ON THE DUALITY METHOD FOR INTEGRO-DIFFERENTIAL EQUATIONS WITH MEASURE DATA
}

\author{
FRANCESCO PETITTA
}

\begin{abstract}
We deal with existence, uniqueness, and regularity for solutions of the boundary value problem

$$
\left\{\begin{array}{l}
\mathcal{L}^{s} u=\mu \quad \text { in } \Omega, \\
u(x)=0 \quad \text { on } \mathbb{R}^{n} \backslash \Omega,
\end{array}\right.
$$

where $\Omega$ is a bounded domain of $\mathbb{R}^{n}, \mu$ is a bounded radon measure on $\Omega$, and $\mathcal{L}^{s}$ is a nonlocal operator of fractional order $s$ whose kernel $K$ is comparable with the one of the factional laplacian.
\end{abstract}

\section{INTRODUCTION}

In the last decade great attention has been paid to the theory of nonlocal operators, in particular integro-differential operators which naturally arise in a huge number of applications as for instance in finance, biology, and physics. The main feature of this type of operators is that they are suitable to be a model to anomalous diffusion process (as for instance Brownian Motions with jumps) or Lévy Processes. For a gentle introduction to these issues we refer to [30, 31] and references therein.

Though the theory of nonlocal operators is nowadays highly developed (e.g. the regularity theory, see for instance [5, 6, 8, and related papers), our aim is to provide a general unified framework to existence, uniqueness and weak regularity for general boundary values problems involving nonlocal operators on bounded domains and highly irregular data (namely measures).

To be more concrete let $\Omega$ be a bounded domain of $\mathbb{R}^{n}$ and let us consider the following boundary value problem

$$
\begin{cases}\mathcal{L}^{s} u=\mu & \text { in } \Omega \\ u(x)=0 & \text { on } \mathbb{R}^{n} \backslash \Omega\end{cases}
$$

where $\mu$ a bounded Radon measure on $\Omega$ and $\mathcal{L}^{s}$ is a nonlocal operator of fractional order $s$, for instance (we will be more precise here below), let $\mathcal{L}^{s}$ be an integrodifferential operator with kernel $K$ such that

$$
K(x, y) \sim \frac{1}{|x-y|^{n+2 s}} .
$$

Following the idea introduced in [16] (and inspired by 27]) for fractional laplacian type problems in $\mathbb{R}^{n}$ we will introduce the notion of duality solutions for problems as (1.1) and we will prove the existence of a unique solution for this problem with minimal assumptions on both the domain and the data.

We want to stress that some of the results we will present here can be found (or deduced) spread in the current literature. In particular, the case $\mathcal{L}^{s}=(-\Delta)^{s}$ (i.e. is the fractional laplacian of order $s$ ) and $\Omega=\mathbb{R}^{n}$, as we already mentioned,

2010 Mathematics Subject Classification. 35R06, 35R09, 35R11, 45K05.

Key words and phrases. Integro-Differential operators, fractional laplacian, measure data, existence, uniqueness, duality solutions. 
was treated in 16, while Dirichlet boundary values problems with smooth data in bounded domains was considered for instance in [10, 22, 23]. Again in the fractional laplacian case, boundary value problems with measure data in smooth domains (namely $C^{2}$ ) was recently considered in 11 .

For more general operators the theory is less complete and, besides the classical books by E. Stein $(28)$ and N. S. Landkof ([20]), we refer the reader to 17, 13 , 14, 21, 4]. We also mention the recent [19] in which the regularity theory for the so called SOLA solutions .

As we said, one of our goals is to provide a common framework for all these previous results: we will indeed consider general nonlocal operators on bounded domains and measure data. We will address the question of existence, uniqueness and weak (e.g. fractional) regularity for solution to Dirichlet problems as in (1.1).

The paper is organized as follows: in Section 2 we give our concept of solution for rather general integro-differential boundary value problems, we investigate the relation of such a concept with the classical weak notion, and we state and prove the existence and uniqueness of a duality solutions. Section 3 will be devoted to the discussion of fractional sobolev regularity for duality solutions as well as further remarks and comments.

\section{General Integro-Differential equations}

In this section we describe and prove our main existence and uniqueness result. As we shall stress later the Stampacchia's duality method we are going to use is robust enough to be applied to very general linear nonlocal operators (see also Remark 2.12 below). Despite of this fact, for the sake of concreteness we will develop our argument in the (anyhow large) class of symmetric integro-differential operators. In particular, for $s \in(0,1)$, we are interested in solving the following Dirichlet boundary value problem:

$$
\left\{\begin{array}{rll}
\mathcal{L}^{s} u & =\mu & \text { in } \Omega \\
u & =0 & \text { in } \mathbb{R}^{n} \backslash \Omega,
\end{array}\right.
$$

where $\mu$ is any bounded radon measure and $\Omega$ is any bounded domain of $\mathbb{R}^{n}, n \geq 2$. The operator $\mathcal{L}^{s}$ is given by

$$
\mathcal{L}^{s} u(x)=\mathrm{PV} \int_{\mathbb{R}^{n}}(u(x)-u(x+y)) K(y) d y,
$$

where $K$ is a nonnegative kernel satisfying

$$
\frac{\lambda}{|y|^{n+2 s}} \leq K(y) \leq \frac{\Lambda}{|y|^{n+2 s}},
$$

with $0<\lambda \leq \Lambda$.

This class of kernels is, for instance, the one considered by Caffarelli-Silvestre in [8. 9] (see also [13, 21]), and it contains as a particular case the fractional laplacian case, that is

$$
(-\Delta)^{s} u(x):=c_{n, s} \mathrm{PV} \int_{\mathbb{R}^{n}} \frac{u(x)-u(x+y)}{|y|^{n+2 s}} d y,
$$

with

$$
c_{n, s}=\frac{4^{s} s(s-1) \Gamma\left(\frac{n+2 s}{2}\right)}{\pi^{\frac{n}{2}} \Gamma(2-s)},
$$

where $\Gamma$ is the Euler Gamma Function.

Here is our notion of solution which extends the one given in [27] (see also [16]). 
Definition 2.1. We say that a function $u \in L^{1}\left(\mathbb{R}^{n}\right)$, is a duality solution for problem (2.2) if $u \equiv 0$ in $\mathbb{R}^{n} \backslash \Omega$ and

$$
\int_{\Omega} u g d x=\int_{\Omega} w d \mu
$$

for all $g \in C_{0}^{\infty}(\Omega)$, where $w$ is the weak solution of

$$
\left\{\begin{array}{rll}
\mathcal{L}^{s} w & =g & \text { in } \Omega \\
w & =0 & \text { in } \mathbb{R}^{n} \backslash \Omega .
\end{array}\right.
$$

Remark 2.2. Some remarks are in order to be done here. First of all the existence of a weak solution (whose definition will be recalled later, see Definition 2.8 below) for problem (2.6) is an easy consequence of Lax-Milgram lemma (see for instance [14] or [21]).

In order for all terms in (2.5) to be well defined we need $w \in C(\bar{\Omega})$ (or, at least, $w \in L^{\infty}(\Omega)$ if $\left.\mu \in L^{1}(\Omega)\right)$; this will be showed in Lemma 2.11 below.

We refer to Section 2.2 below for further comments on this definition, the relations with the other available definitions and to the sense in which the boundary data is assumed.

Before stating our main result we need to recall the following definition

Definition 2.3. We say that $\Omega$ satisfies the uniform exterior ball condition if there exists $s_{\Omega}>0$ such that for every $x \in \mathbb{R}^{n} \backslash \Omega$ with $\operatorname{dist}(x, \partial \Omega)<s_{\Omega}$, there is $z_{x} \in \partial \Omega$ such that $\left|x-z_{x}\right|=\operatorname{dist}(x, \partial \Omega)$ and $B\left(x_{0}, s_{\Omega}\right) \subset \mathbb{R}^{n} \backslash \Omega$ with $x_{0}:=z_{x}+s_{\Omega} \frac{x-z_{x}}{\left|x-z_{x}\right|}$. In an analogous way, one can define the uniform interior ball condition by replacing $\mathbb{R}^{n} \backslash \Omega$ with $\Omega$.

In order to better understand the previous definition, let us recall that, as is proved in [2, Corollary 3.14], a domain with compact boundary is of class $\mathcal{C}^{1,1}$ if and only if it satisfies both a uniform interior ball condition and an exterior one.

Here is our existence and uniqueness result

Theorem 2.4. Let $\Omega$ be a bounded open set satisfying the uniform exterior ball condition. Then there exists a unique duality solution for problem (2.2) in the sense of Definition 2.1. Moreover, $u \in L^{q}\left(\mathbb{R}^{n}\right)$, for any $q<\frac{n}{n-2 s}$.

Remark 2.5. As we will deduce from the proof of Theorem 2.4 the regularity condition on $\Omega$ is essential in order to get continuity estimates up to the boundary for the solution. In the case of a general bounded domain, if $\mu \in L^{1}(\Omega)$, with the same proof, one can obtain existence and uniqueness of a duality solution for problem (2.2) which turns out to coincide with the one obtained in 21.

Also observe that, as already noticed in [16] for the solutions in $\mathbb{R}^{n}$, the regularity of the solution we find is optimal as the fundamental solution (i.e. the solution with datum $\mu=\delta_{0}$ ) for these operators is comparable to $|x|^{2 s-n}$ near the origin. Finally observe that, as $s$ goes to $1^{-}$, we formally obtain the sharp regularity of [27 for boundary value problems involving linear second order differential equations. This latter fact is completely formal as, as $s$ approaches 1 nonlocal integro-differential operators may degenerate.

2.1. Some useful notations and tools. We will made use of some basic results concerning fractional Sobolev spaces (also called spaces of Bessel potentials). For a review in the subject we refer to [1, 28, (see also [12]). Let us recall the following

Definition 2.6. For $0<s<1$ and $1 \leq p<\infty$. We define the fractional sobolev space $W^{s, p}\left(\mathbb{R}^{n}\right)$ as the set of all functions $u$ in $L^{p}\left(\mathbb{R}^{n}\right)$ such that

$$
\int_{\mathbb{R}^{n}} \int_{\mathbb{R}^{n}} \frac{|u(x)-u(y)|^{p}}{|x-y|^{n+s p}} d x d y<\infty,
$$


endowed with the norm

$$
\|u\|_{W^{s, p}\left(\mathbb{R}^{n}\right)}=\|u\|_{L^{p}\left(\mathbb{R}^{n}\right)}+\left(\int_{\mathbb{R}^{n}} \int_{\mathbb{R}^{n}} \frac{|u(x)-u(y)|^{p}}{|x-y|^{n+s p}} d x d y\right)^{\frac{1}{p}} .
$$

If $p=2$ we will use the usual notation $W^{s, p}\left(\mathbb{R}^{n}\right)=H^{s}\left(\mathbb{R}^{n}\right)$.

If $\Omega$ is any bounded domain of $\mathbb{R}^{n}$ the space $W^{s, p}(\Omega)$ is defined in a similar way, while $W_{0}^{s, p}(\Omega)$ is defined as the closure of $C_{0}^{\infty}(\Omega)$ with respect to the norm defined in (2.7).

The following embedding theorem is valid in domains satisfying the so-called extension property which, roughy speaking, consists in the fact that functions in $W^{s, p}(\Omega)$ can be extended to functions in $W^{s, p}\left(\mathbb{R}^{n}\right)$. Concretely one can think, for instance, of $\Omega$ to be an open domain with lipschitz boundary.

We recall the fractional Sobolev embedding result we shall use (see [1]).

Theorem 2.7 (Sobolev embedding). Let $\Omega$ be a bounded domain of $\mathbb{R}^{n}$ with the extension property and let $s p<n$. Then, there exists a constant $C$ depending only on $s$ and $n$, such that

$$
\|v\|_{L^{p_{s}^{*}}(\Omega)} \leq C\|v\|_{W^{s, p}(\Omega)}, \quad \forall v \in W^{s, p}(\Omega),
$$

where $p_{s}^{*}=\frac{n p}{n-s p}$ is the fractional Sobolev critical exponent, $p>1$ and $0<s<1$.

Moreover, if $\Omega$ has no external cusps and $p s>n$, then

$$
\|v\|_{C^{\gamma}(\Omega)} \leq C\|v\|_{W^{s, p}(\Omega)}, \quad \forall v \in W^{s, p}(\Omega),
$$

where $\gamma=\frac{s p-n}{p}$.

2.2. Weak solution vs Duality Solution. Due to the generality of the results we presented, some important features of the duality formulation we introduced could be be missed. At first glance, in such a nonregular framework, one could ask weather the duality formulation is the good one in order to give sense to the boundary datum $u=0$ on $\mathbb{R}^{n} \backslash \Omega$.

In order to give some insights towards the question of the boundary datum we restrict ourself to the toy model of the fractional laplacian case. The reader will easily deduce that the following arguments keep working in more general cases as an integration by parts formula in all of $\mathbb{R}^{n}$ is available for functions in $H^{s}\left(\mathbb{R}^{n}\right)$.

Let $\Omega$ be a bounded open set of $\mathbb{R}^{n}$ and let us consider the following problem

$$
\left\{\begin{array}{l}
(-\Delta)^{s} u=\mu \quad \text { in } \Omega \\
u(x)=0 \quad \text { on } \quad \mathbb{R}^{n} \backslash \Omega
\end{array}\right.
$$

where $\mu$ is a bounded Radon measure on $\Omega$ of $\mathbb{R}^{n}$, and $(-\Delta)^{s}$ is the fractional laplace operator introduced in (2.4) with $s \in(0,1)$.

As we already noticed, for data regular enough (namely if $\mu \in H^{-s}(\Omega)$ ), then existence of a finite energy solution (i.e. weak solutions in $H^{s}(\Omega)$ ) for problem (2.8) is an easy consequence of Lax-Milgram Lemma (see for instance [21]). Existence, uniqueness and regularity up to the boundary for solutions to problem (2.8) can be found in $\left[22\right.$ in the case of bounded data $\mu \in L^{\infty}(\Omega)$.

In Definition 2.1 we added the boundary condition $u=0$ on $\mathbb{R}^{n} \backslash \Omega$. This seems to be quite artificial and it need to be better explained. If $u$ satisfies (2.5) then, at 
least formally, using the integration by parts formula in all $\mathbb{R}^{n}$, we have

$$
\begin{aligned}
\int_{\Omega} w d \mu=\int_{\Omega} u(-\Delta)^{s} w & =\int_{\mathbb{R}^{n}} u(-\Delta)^{s} w-\int_{\mathbb{R}^{n} \backslash \Omega} u(-\Delta)^{s} w \\
& =\int_{\mathbb{R}^{n}}(-\Delta)^{s} u w-\int_{\mathbb{R}^{n} \backslash \Omega} u(-\Delta)^{s} w \\
& =\int_{\Omega} w(-\Delta)^{s} u-\int_{\mathbb{R}^{n} \backslash \Omega} u(-\Delta)^{s} w
\end{aligned}
$$

where in the last equality we have used $w \equiv 0$ in $\mathbb{R}^{n} \backslash \Omega$.

The previous identity can be recast as

$$
\int_{\Omega} w d \mu=\int_{\Omega} w(-\Delta)^{s} u+\int_{\Omega} \int_{\mathbb{R}^{n} \backslash \Omega} \frac{w(x) u(y)}{|x-y|^{n+2 s}} d x d y .
$$

In particular (2.9) is satisfied for all $w \in C_{c}^{\infty}(\Omega)$. That is,

$$
(-\Delta)^{s} u(x)+\int_{\mathbb{R}^{n} \backslash \Omega} \frac{u(y)}{|x-y|^{n+2 s}} d y=\mu(x), \text { in } \mathcal{D}^{\prime}(\Omega) .
$$

The second term is a function of $x$ that depends on the values of $u$ outside. This suggest to impose $u=0$ on $\mathbb{R}^{n} \backslash \Omega$. Proposition 2.9 below clarifies that this is the right choice. First of all we need the following definition (see [22]) recalling that, by Theorem 2.7, $L^{\left(2_{s}^{*}\right)^{\prime}}(\Omega) \subset H^{-s}(\Omega)$.

Definition 2.8. Let $\mu \in L^{\left(2_{s}^{*}\right)^{\prime}}(\Omega)$. A weak solution for problem (2.8) is a function $u \in H^{s}\left(\mathbb{R}^{n}\right)$ such that $u \equiv 0$ a.e. on $\mathbb{R}^{n} \backslash \Omega$, and

$$
\int_{\mathbb{R}^{n}}(-\Delta)^{\frac{s}{2}} u(-\Delta)^{\frac{s}{2}} v d x=\int_{\Omega} \mu v d x
$$

for any $v \in H^{s}\left(\mathbb{R}^{n}\right)$ such that $v \equiv 0$ (a.e.) on $\mathbb{R}^{n} \backslash \Omega$.

Proposition 2.9. Let $\mu \in L^{\left(2_{s}^{*}\right)^{\prime}}(\Omega)$ then $u$ is a duality solution of problem (2.8) in the sense of Definition 2.1 if and only if $u$ is a weak solution of problem (2.8).

Proof. Let $u$ be a weak solution of problem (2.8), and let $g \in C_{0}^{\infty}(\Omega)$. If $w$ be the corresponding solution with datum $g$. Then, integrating by parts and using that $u=0$ outside $\Omega$, we have

$$
\begin{aligned}
& \int_{\Omega} u g d x=\int_{\Omega} u(-\Delta)^{s} w d x=\int_{\mathbb{R}^{n}} u(-\Delta)^{s} w d x \\
& -\int_{\mathbb{R}^{n} \backslash \Omega} u(-\Delta)^{s} w d x=\int_{\mathbb{R}^{n}}(-\Delta)^{\frac{s}{2}} u(-\Delta)^{\frac{s}{2}} w d x=\int_{\Omega} \mu w d x .
\end{aligned}
$$

Now, let $u$ be the duality solution of problem (2.8) and let $\tilde{u}$ be the weak solution of the same problem. Then, reasoning as before, we have

$$
\int_{\Omega} \tilde{u} g d x=\int_{\Omega} \mu w d x
$$

for any $g \in C_{0}^{\infty}(\Omega)$. Then, subtracting the formulation of $u$ we get

$$
\int_{\Omega}(u-\tilde{u}) g d x=0
$$

for any $g \in C_{0}^{\infty}(\Omega)$, that implies $u=\tilde{u}$.

Remark 2.10. The previous result shows the equivalence between the duality and the weak formulations in the case of finite energy solutions for homogeneous 
problems in bounded domains. In the case of general nonhomogeneous integrodifferential problems of the form

$$
\left\{\begin{array}{rll}
\mathcal{L}^{s} u & =0 & \text { in } \Omega \\
u & =\phi & \text { in } \mathbb{R}^{n} \backslash \Omega
\end{array}\right.
$$

it is worth introducing the further notion of viscosity solution at least for smooth data $\phi$ (see for instance [8, 3]).

For instance, let $\phi$ be a bounded function in $C^{\gamma}\left(\mathbb{R}^{n} \backslash \bar{\Omega}\right)$ for some positive $\gamma<1$, and let $\Omega$ satisfying the uniform exterior ball condition. The existence of a unique viscosity solution for problem (2.10) can be proved by Perron's method in a standard way through the construction of suitable barriers (see 9] and [18). As pointed out in 22 (see also 25) the unique viscosity solution for problem (2.10) turns out to coincides with the weak one due to both the interior regularity for viscosity solutions (see for instance 8]) and the existence and uniqueness of weak solution for the same problem (see for instance [14]).

2.3. Existence and Uniqueness of a duality solution. Before proving Theorem 2.4 we need a preliminary result which is based on the main result in [13. In this paper, the authors proved that if

$$
\mathcal{L}^{s} v=h \quad \text { in } \mathbb{R}^{n},
$$

and $h \in L^{p}\left(\mathbb{R}^{n}\right)$, with $p>1$, then

$$
\|v\|_{W^{2 s, p}\left(\mathbb{R}^{n}\right)} \leq C\|h\|_{L^{p}\left(\mathbb{R}^{n}\right)} .
$$

By the Sobolev embedding, this implies that

$$
\|v\|_{C \gamma\left(\mathbb{R}^{n}\right)} \leq C\|h\|_{L^{p}\left(\mathbb{R}^{n}\right)} \quad \text { whenever } p>\frac{n}{2 s} .
$$

Lemma 2.11. Let $\Omega$ be any bounded domain, $p>\frac{n}{2 s}, f \in L^{p}(\Omega)$, and $u$ be $a$ solution of (2.8) with $\mu=f$. Then,

(a) $u$ is bounded and

$$
\|u\|_{L^{\infty}(\Omega)} \leq C\|f\|_{L^{p}(\Omega)} .
$$

(b) If in addition $\Omega$ satisfies the uniform exterior ball condition, then $u$ is $C(\bar{\Omega})$.

Proof. Without loss of generality, we assume that $f \geq 0$ (if not, using linearity we can consider $|f|$ and then apply the result).

Let $\tilde{u}$ be the nonnegative weak solution in all of $\mathbb{R}^{n}$ of

$$
\mathcal{L}^{s} \tilde{u}=f \chi_{\Omega} \quad \text { in } \mathbb{R}^{n} .
$$

By (2.11), we have

where $\gamma=2 s-\frac{n}{p}$.

$$
\|\tilde{u}\|_{C^{\gamma}\left(\mathbb{R}^{n}\right)} \leq C\|f\|_{L^{p}(\Omega)},
$$

Let us now consider $v=\tilde{u}-u$. This function satisfies

$$
\left\{\begin{array}{rll}
\mathcal{L}^{s} v & =0 & \text { in } \Omega \\
v & =\tilde{u} & \text { in } \mathbb{R}^{n} \backslash \Omega
\end{array}\right.
$$

with $\tilde{u} \geq 0$ and $\tilde{u} \in C^{\gamma}\left(\mathbb{R}^{n}\right)$. Let us prove now (a) and (b)

(a) By the maximum principle, since $\tilde{u} \geq 0$ then we will have $v \geq 0$. Thus,

$$
0 \leq u=\tilde{u}-v \leq \tilde{u} \leq C\|f\|_{L^{p}(\Omega)},
$$

and hence

$$
\|u\|_{L^{\infty}(\Omega)} \leq C\|f\|_{L^{p}(\Omega)} .
$$

(b) As $\Omega$ satisfies the uniform exterior ball condition and $\tilde{u}$ is $C^{\gamma}\left(\mathbb{R}^{n}\right) \cap L^{\infty}\left(\mathbb{R}^{n}\right)$, then $v$, the solution of (2.12), is $C(\bar{\Omega})$ (see 9], [18, and Remark 2.10 above).

This concludes the proof of Lemma 2.11 as $u=\tilde{u}-v$. 
Proof of Theorem 2.4. In order to get the optimal regularity of $u$ we will consider less regular functions $g$ in Definition 2.1. The equivalence between the two definition relies on a straightforward density argument. Let us fix $p>\frac{n}{2 s}$. For any $g \in L^{p}(\Omega)$, let us define the following operator $T: L^{p}(\Omega) \mapsto \mathbb{R}$ through

$$
T(g):=\int_{\Omega} w(x) d \mu .
$$

Using Lemma 2.11, $T$ is well defined, and we can write

$$
|T(g)| \leq\|w\|_{L^{\infty}(\Omega)}|\mu|(\Omega) \leq C\|g\|_{L^{p}(\Omega)},
$$

where $C$ depends only on $\Omega, \mu, n, s$ and $p$. Then $T$ is a bounded continuous linear functional on $L^{p}(\Omega)$, so that by Riesz Representation Theorem, there exists a unique function $u \in L^{p^{\prime}}(\Omega)$, such that

$$
\int_{\Omega} w d \mu=\int_{\Omega} u g
$$

Of course, we can repeat the argument for any $p>\frac{n}{2 s}$ and we find a unique $u \in L^{p^{\prime}}(\Omega)$ (and so $p^{\prime}<\frac{n}{n-2 s}$ ) such that (2.13) holds.

Uniqueness easily follows by the fundamental theorem of calculus of variations as if $u$ and $v$ are two solutions in the sense of Definition 2.1 then one has

$$
\int_{\Omega}(u-v) g=0
$$

for any $g \in C_{0}^{\infty}(\Omega)$ and so $u=v$.

Remark 2.12. Let us notice the fact that our existence and uniqueness result for these general integro-differential operators does not rely at all on the knowledge of the fundamental solution. Moreover, continuity of solution to problem (2.8) with sufficiently smooth data is what is needed here in order to apply the duality method. However, more precise regularity results up to the boundary can be found in the literature in some particular cases: e.g. the fractional laplace case $([22])$ or the case of anisotropic $\alpha$-stable processes ([24], see also Section 3.1 below for a precise definition of these latter type of operators).

We also would like to stress a general fact about the method we used. As it is well known (and, as one can easily deduce from the proof of Theorem 2.4) the Stampacchia's duality method relies essentially on two main ingredients: linearity (in particular on the possibility to define an adjoint operator, $\mathcal{L}^{s}$ itself in Theorem 2.4 as $\mathcal{L}^{s}$ is self-adjoint), and a regularity result. For the sake of exposition we restricted ourself on the integro-differential case with symmetric kernels. Anyhow, it is clear that a formally identical existence and uniqueness metatheorem can be proved in the same way for very general boundary value problems involving (local or) nonlocal linear operators. For a review on more general nonlocal operators (e.g. $x$-dependent kernels, non-symmetric case, etc.) we refer for instance to 14 and references therein. Roughly speaking, such a metatheorem reads as: let $\mathcal{L}$ be a nonlocal linear operator, $\mu$ a bounded radon measure on $\Omega$, and let $\mathcal{G}^{*}$ be the Green operator for the homogeneous Dirichlet boundary value problem associated to the adjoint operator $\mathcal{L}^{*}$. If $\mathcal{G}^{*}$ maps continuously $L^{q}(\Omega)$ into $C(\bar{\Omega})$, for any $q>q_{0}$, then there exist a unique duality solution for $u \in L^{p}(\Omega)$ for any $p<\frac{q_{0}}{q_{0}-1}$ for

$$
\left\{\begin{array}{l}
\mathcal{L} u=\mu \quad \text { in } \Omega, \\
u=0 \quad \text { on } \quad \mathbb{R}^{n} \backslash \Omega .
\end{array}\right.
$$




\section{Further Remarks And Extensions}

This final section is devoted to present some further remarks on the regularity of the duality solutions found in Theorem 2.4. One of the main tools in order to study Sobolev fractional regularity for solutions to Dirichlet integro-differential problems relies on the use of Bessel potentials associated with this kind of operators. A general treatment of this issue is out of the purpose of this note so, for the sake of concreteness, we will describe the method in some particular cases.

3.1. Local regularity for $\alpha$-stable processes. As one can imagine Sobolev fractional regularity of duality solutions can be deduced if we have some informations on the exact behavior of the fundamental solution for this operator. For the sake of exposition we consider the case of anisotropic $\alpha$-stable process and we readapt the result in 16 in order to get sharp local Sobolev regularity for the solution of (2.8).

Let $\Omega$ be a bounded open set of $\mathbb{R}^{n}$. Consider

$$
\left\{\begin{array}{rll}
L^{\alpha} u & =\mu & \text { in } \Omega \\
u & =0 & \text { in } \mathbb{R}^{n} \backslash \Omega,
\end{array}\right.
$$

where $\mu$ is a radon bounded measure on $\Omega$. The operator $L^{\alpha}$ is given by

$$
L^{\alpha} u(x)=\mathrm{PV} \int_{\mathbb{R}^{n}}(u(x)-u(x+y)) \frac{a(y /|y|)}{|y|^{n+\alpha}} d y,
$$

where $0<\alpha<2$ and $a: S^{n-1} \longrightarrow \mathbb{R}$ is a nonnegative and symmetric function that satisfies the uniform ellipticity condition

$$
\lambda \leq a(\theta) \leq \Lambda \quad \text { for all } \quad \theta \in S^{n-1},
$$

with $0<\lambda \leq \Lambda$.

These operators are infinitesimal generators of a very special class of Lévy processes: the so-called $\alpha$-stable processes. With respect to the previous section here we use the usual convention $\alpha=2 s$

It is proved in [29] that the potential kernel $K$ associated to $L^{\alpha}$ (i.e., the fundamental solution of the operator) satisfies

$$
\frac{c_{1}}{|y|^{n-\alpha}} \leq K(y) \leq \frac{c_{2}}{|y|^{n-\alpha}}
$$

for suitable positive constants $c_{1} \leq c_{2}$, and

$$
K(y)=|y|^{\alpha-n} K\left(\frac{y}{|y|}\right) .
$$

Moreover, by Theorem 1 in [7, we have that $K$ belongs to the Hölder space $C_{\text {loc }}^{2 \alpha-\epsilon}\left(\mathbb{R}^{n} \backslash\{0\}\right)$ for all $\epsilon>0$.

Thus, it follows that $K$ is a fractional kernel of order $\alpha$ and regularity $2 \alpha-\epsilon$ in the sense of Definition 4.1 in [15. In particular, we have the following

Theorem 3.1. The Riesz potential associated to $L^{\alpha}$

$$
I^{\alpha}(f)(x)=\int_{\mathbb{R}^{n}} f(y) K(x-y) d y
$$

maps $L^{p}\left(\mathbb{R}^{n}\right)$ boundedly into $C^{\alpha-\frac{n}{p}}\left(\mathbb{R}^{n}\right)$ whenever $p>n / \alpha$. That is,

$$
\left\|I^{\alpha}(f)\right\|_{C^{\alpha-\frac{n}{p}}\left(\mathbb{R}^{n}\right)} \leq C\|f\|_{L^{p}\left(\mathbb{R}^{n}\right)},
$$

with $C$ depending only on $p, n, \lambda, \Lambda$, and $\alpha$.

Proof. see Theorem 5.2 in [15]. 
Due to Theorem 3.1, and in particular to the integral representation 3.15 it is straightforward to reproduce the argument in [16] in order to show the existence of a unique duality solution $\tilde{u}$ for problem

$$
L^{\alpha} u=\mu \text { in } \mathbb{R}^{n}
$$

whenever $\mu$ is set to be zero outside $\Omega$. Moreover, $\tilde{u} \in W_{l o c}^{1-\frac{2-\alpha}{q}, q}\left(\mathbb{R}^{n}\right)$ for any $q<\frac{n+2-\alpha}{n+1-\alpha}$

For simplicity, in what follows we also assume $\alpha>1$, (i.e. $s>\frac{1}{2}$, see [16, Pag. 2 , and Remark 3.4 below for further comments on this restriction) and $\mu$ to be nonnegative.

We have the following local result

Theorem 3.2. The duality solution $u$ of problem 3.14 belongs to $W_{l o c}^{1-\frac{2-\alpha}{q}, q}(\Omega)$ for any $q<\frac{n+2-\alpha}{n+1-\alpha}$.

Proof. Let $\tilde{u}$ be the duality solution of

$$
L^{\alpha} \tilde{u}=\mu \quad \text { in } \quad \mathbb{R}^{n},
$$

where, as we said, $\mu$ is extended by zero outside $\Omega$. Let us consider now $v:=\tilde{u}-u$. This function is a nonnegative function in all of $\mathbb{R}^{n}$, and it satisfies $(-\Delta)^{s} v=0$ in $\Omega$. Moreover, $v=\tilde{u}$ outside $\Omega$, and in particular $\tilde{u} \in L^{\infty}\left(\mathbb{R}^{n} \backslash \Omega\right)$. that implies that $v$ is locally smooth inside $\Omega$ (see for instance [26, 8]). So that, $v$ also satisfies that $v \in W_{l o c}^{1-\frac{2-\alpha}{q}, q}(\Omega)$ for any $q<\frac{n+2-\alpha}{n+1-\alpha}$, and hence the proof is complete as $u=\tilde{u}-v$.

3.2. Towards a global regularity result. Let us come back to general integrodifferential Dirichlet boundary value problem

$$
\left\{\begin{array}{rll}
\mathcal{L}^{s} u & =\mu & \text { in } \Omega \\
u & =0 & \text { in } \mathbb{R}^{n} \backslash \Omega
\end{array}\right.
$$

where $\mathcal{L}^{s}$ is of the type we considered in Section 2. As we already said, a fine study of the Bessel potentials associated with these operators is out of our scopes here, so, for the sake of presentation, we are going to straighten the assumptions on both the operators $\mathcal{L}^{s}$ and on the admissible domains $\Omega$. We assume that $\mathcal{L}^{s}$ satisfies the following Calderón-Zygmund type property: for $\nu \geq 0$ and $r>N+2-2 s$, there exist $C>0$ such that,

$$
\left\|\mathcal{L}^{-s} g\right\|_{W^{2 s-\nu, r}(\Omega)} \leq C\|g\|_{W^{-\nu, r}(\Omega)}
$$

for any $g \in W^{-\nu, r}(\Omega)$, where $\mathcal{L}^{-s} g$ is the solution of

$$
\left\{\begin{array}{rll}
\mathcal{L}^{s} w= & \text { in } \Omega \\
w=0 & \text { in } \mathbb{R}^{n} \backslash \Omega .
\end{array}\right.
$$

Properties as (3.17), which are established for instance in the fractional laplace case, are natural for general operators of fractional order $s$ at least for sufficiently smooth domain (see for instance [32, 13]).

Concerning the regularity of the domain, for simplicity we may think at $\partial \Omega \in$ $\mathcal{C}^{1,1}$. Anyway, this assumption is not sharp. The same argument will work for more general bounded domains of $\mathbb{R}^{n}$ satisfying the extension property, the uniform ball condition and with no external cusps. Moreover, as the proof will essentially be based on Sobolev embeddings, if $\mu \in L^{1}(\Omega)$ then one can remove the exterior ball assumption on $\Omega$ (e.g. Lipschitz domains).

Theorem 3.3. Let $\mathcal{L}^{s}$ and $\Omega$ as above, then the duality solution of problem (3.16) belongs to $u \in W_{0}^{\eta, q}(\Omega)$, for any $q<\frac{n+2-2 s}{n+1-2 s}$, where $\eta=1-\frac{2-2 s}{q}$. 
Remark 3.4. We notice that the derivation exponent $\eta=1-\frac{2-2 s}{q}$ can be negative. This is not the case if, for instance, $s>\frac{1}{2}$, so, for the sake of exposition we will understand this assumption in the following proof. However, the result is formally correct for any $s \in(0,1)$ once one interpret the space $W^{\eta, q}(\Omega)$ in the distributional sense as the dual of $W_{0}^{-\eta, q^{\prime}}(\Omega)$ (see again [12]).

Finally, we want to emphasize that, as $s \rightarrow 1^{-}$, we formally recover the classical optimal summability of the gradient for linear elliptic boundary value problems with measure data (see again [27]), $u \in W_{0}^{1, q}(\Omega)$, for any $q<\frac{n}{n-1}$. Also notice that, as before, the result is optimal, as it coincides with the regularity of the fundamental solution for the fractional Laplacian $G(x)=c|x|^{2 s-n}$.

Proof. Let us fix $q$ as in the statement of Theorem 3.3 and let $\eta=1-\frac{2-2 s}{q}$. Observe that $(2 s-\eta) q^{\prime}>n$ if and only if $q<\frac{n+2-2 s}{n+1-2 s}$. In particular, under this assumption we can use Theorem 2.7 in order to have

$$
\|w\|_{L^{\infty}(\Omega)} \leq C\|w\|_{W^{2 s-\eta, q^{\prime}}(\Omega)}, \quad \forall w \in W^{2 s-\eta, q^{\prime}}(\Omega),
$$

Hence, we reason as in the proof of Theorem 2.4. and, using (3.17), we have that, for any $g \in C_{0}^{\infty}(\Omega)$

$$
|T(g)| \leq\|w\|_{L^{\infty}(\Omega)}|\mu|(\Omega) \leq C\|w\|_{W^{2 s-\eta, q^{\prime}}(\Omega)} \leq C\|g\|_{W^{-\eta, q^{\prime}}(\Omega)},
$$

from which we deduce that $T$, defined as

$$
T(g):=\int_{\Omega} w(x) d \mu=\int_{\Omega} u g,
$$

is a linear and continuous operator on $W^{-\eta, q^{\prime}}(\Omega)$, that implies $u \in W_{0}^{\eta, q}(\Omega)$ as $u \equiv 0$ outside of $\Omega$.

Acknowledgement. The author is deeply grateful to Xavier Ros-Oton for essential advices and fruitful discussions during the preparation of this manuscript. The author is partially supported by the Gruppo Nazionale per l'Analisi Matematica, la Probabilità e le loro Applicazioni (GNAMPA) of the Istituto Nazionale di Alta Matematica (INdAM).

\section{REFERENCES}

1. R. A. Adams and J. J. F. Fournier. Sobolev Spaces. Second edition (Academic Press, 2003).

2. R. Alvarado, D. Brigham, V. Maz'ya, M. Mitrea and E. Ziadé. On the regularity of domains satisfying a uniform hour-glass condition and a sharp version of the Hopf-Oleinik boundary point principle. J. Math. Sciences. 176 (2011), 281-360.

3. G. Barles, E. Chasseigne and C. Imbert. On the Dirichlet problem for second-order elliptic integro-differential equations, Indiana Univ. Math. J. 57 (2008), 213-246.

4. B. Barrios, I. Peral, S. Vita, Some remarks about the summability of nonlocal nonlinear problems, Advances in Nonlinear Analysis, to appear

5. M. T. Barlow, R. F. Bass, Z-Q. Chen and M. Kassmann. Non-local Dirichlet forms and symmetric jump processes. Trans. Amer. Math. Soc. 361 (2009), 1963-1999.

6. R. F. Bass. Regularity results for stable-like operators. J. Funct. Anal. 257 (2009), 2693-2722.

7. K. Bogdan, A. Stós and P. Sztonyk. Potential theory for Lévy stable processes. Bull. Polish Acad. Sci. Math. 50 (2002), 361-372.

8. L. Caffarelli and L. Silvestre. Regularity theory for fully nonlinear integro-differential equations. Comm. Pure Appl. Math. 62 (2009), 597-638.

9. L. Caffarelli and L. Silvestre. Regularity results for nonlocal equations by approximation. Archive Rational Mech. Anal. 200 (2011), 59-88.

10. Z-Q. Chen and R. Song. Estimates on Green functions and Poisson kernels for symmetric stable processes. Math. Ann. 312, (1998), 465-501.

11. H. Chen and L. Veron. Semilinear fractional elliptic equations involving measures. arXiv: $1305.0945 \times 2$

12. E. Di Nezza, G. Palatucci and E. Valdinoci. Hitchhiker's guide to the fractional Sobolev spaces. Bull. Sci. Math. 136 (2012), 521-573. 
13. H. Dong and D. Kim. On $L^{p}$ estimates for a class of nonlocal elliptic equations. J. Funct. Anal. 262 (2012), 1166-1199.

14. M. Felsinger, M. Kassmann and P. Voigt. The Dirichlet problem for nonlocal operators. arXiv:1309.5028v2

15. J. García-Cuervo and E. Gatto. Boundedness properties of fractional integral operators associated to non-doubling measures. Publ. Mat. 49 (2005), 285-296.

16. K. H. Karlsen, F. Petitta and S. Ulusoy. A duality approach to the fractional laplacian with measure data. Publ. Mat. 55 (2011), 151-161.

17. M. Kassmann. A priori estimates for integro-differential operators with measurable kernels. Calc. Var. Partial Differential Equations. 34 (2009), 1-21.

18. Y-C. Kim and K.-A. Lee. Regularity Results for Fully Nonlinear Integro-Differential Operators with Nonsymmetric Positive Kernels: Subcritical Case. Potential Anal. 38 (2013), 433-455.

19. T. Kuusi, G. Mingione, Y. Sire. Nonlocal equations with measure data. Comm. Math. Phys., to appear

20. N. S. Landkof, Foundations of modern potential theory, (Berlin: Springer-Verlag, 1972).

21. T. Leonori, I. Peral, A. Primo and F. Soria. Basic estimates for solutions of a class of nonlocal elliptic and parabolic equations. Discrete and Continuous Dynamical System, to appear

22. X. Ros-Oton and J. Serra. The Dirichlet problem for the fractional laplacian: regularity up to the boundary. J. Math. Pures Appl. 101 (2014), 275-302.

23. X. Ros-Oton and J. Serra. The extremal solution for the fractional Laplacian. Calc. Var. Partial Differential Equations. In press.

24. X. Ros-Oton and J. Serra. Boundary regularity for fully nonlinear integro differential equations. Preprint, 2014.

25. R. Servadei and E. Valdinoci. Weak and viscosity solutions of the fractional Laplace equation. Publ. Mat. 58 (2014), 133-154.

26. L. Silvestre. Regularity of the obstacle problem for a fractional power of the Laplace operator. PhD thesis. (University of Texas at Austin, 2005).

27. G. Stampacchia. Le problème de Dirichlet pour les équations elliptiques du seconde ordre à coefficientes discontinus. Ann. Inst. Fourier (Grenoble). 15 (1965), 189-258.

28. E. Stein. Singular Integrals and Differentiability Properties of Functions. (New York: Princeton Univ. Press, 1970).

29. P. Sztonyk. Regularity of harmonic functions for anisotropic fractional Laplacian. Studia Math. 181 (2007), 101-123.

30. E. Valdinoci. From the long jump random walk to the fractional Laplacian. Bol. Soc. Esp. Mat. Apl. SeMA. 49 (2009), 33-44.

31. J. L. Vazquez. Nonlinear diffusion with fractional laplacian operators. In Nonlinear partial differential equations: the Abel Symposium 2010 (Holden, Helge and Karlsen, Kenneth H. eds.) pp. 271-298 (Springer, 2012).

32. W. Ziemer. Weakly Differentiable Functions. (Berlin: Springer-Verlag, 1989).

(F. Petitta) Dipartimento di Scienze di Base e Applicate per L' Ingegneria, "Sapienza", Università di Roma, Via Scarpa 16, 00161 Roma, Italy.

E-mail address: francesco.petitta@sbai.uniroma1.it 